\title{
Alpha Oscillations underlie Working Memory Abnormalities in the Psychosis High-risk State
}

Avinash Ramyead ${ }^{1,2}, \mathrm{PhD}$; Michael Kometer ${ }^{3}, \mathrm{PhD}$; Erich Studerus ${ }^{1}, \mathrm{PhD}$; Denise Baumeler ${ }^{1}$, BA; Robin von Rotz ${ }^{1}$, BA; Anita Riecher-Rössler ${ }^{1 *}$, MD

${ }^{1}$ University of Basel Psychiatric Clinics, Center for Gender Research and Early Detection, Basel, Switzerland

${ }^{2}$ University of California, San Francisco, Department of Psychiatry, San Francisco, USA

${ }^{3}$ Neuropsychopharmacology and Brain Imaging Research Unit, Department of Psychiatry, Psychotherapy and Psychosomatics, Hospital of Psychiatry, University of Zurich, Switzerland

Running title: Working memory in the psychosis high-risk state

*Corresponding author:

Prof. Anita Riecher-Rössler, MD

University of Basel Psychiatric Clinics

Center for Gender Research and Early Recognition

Kornhausgasse 7

CH-4051 Basel, Switzerland

Phone: +41613258161

Fax: +41613258160

E-mail: anita.riecher@upkbs.ch

Word count in Abstract: 150

Word count in article body: 3400 
Ramyead et al., 2016

\begin{abstract}
Working memory (WM) functioning, known to be modulated by neural oscillations, is impaired in schizophrenic psychoses. It remains unclear whether in the psychosis high-risk state, WM encoding is altered or whether patients are impaired at shielding their WM against distractors. We employed single-trial analyses of neurophysiological and behavioral data recorded during a WM paradigm, designed to include predictable distractors, on 18 patients with an at-risk mental state for psychosis (ARMS,26.1+/-5.45years) and 21 healthy controls(HCs,25.5+/-3.95years). Strong distractors were associated with reduced WM accuracy $(p=0.036)$, but only ARMS patients required more processing time for strong distractors $(p=0.002)$. Increased parieto-occipital alpha amplitude preceding distractor presentations was associated with enhanced accuracy only in $\mathrm{HCs}(\mathrm{p}=0.009)$. During encoding, increased intertrial alpha phase locking values were associated with increased performance. Reduced shielding mechanisms against distractors in ARMS patients could lead to defective WM maintenance, which may result in significant confusion that may contribute to the formation of psychotic symptoms.
\end{abstract}

Keywords: neural oscillations; EEG; psychosis; schizophrenia; working memory; phase locking 


\section{Introduction}

In an environment in which individuals are continuously bombarded with sensorial stimuli, it is essential to have effective mechanisms for filtering out irrelevant information $(1,2)$. These mechanisms could be partially implemented through alpha oscillations, which are known to be involved in gating of incoming information (3-6), and thereby preserve proper functioning of working memory (WM) (7-9). WM functioning, as well as the ability to gate sensory information, have repeatedly been shown to be impaired in schizophrenic psychoses $(10,11)$, suggesting that an alteration in alpha-oscillations could be a key mechanism in the pathogenesis of schizophrenic psychoses.

Over the past 20 years, numerous studies have assessed patients with a clinical high risk of developing psychosis to identify key mechanism in the pathogenesis of schizophrenia that would allow for an early intervention. These patients with an at-risk mental state (ARMS) for psychosis eventually develop psychosis within 3 years with a probability of about $36 \%(12,13)$. These patients were found to have decreased P50 suppression in the sensory-gating ERP paradigm (14), suggesting that they have a poor "shielding" against internal noise and external distractors (1). That is, these patients could be highly sensitive to distractors, which would prevent them from having a well-functioning and stable WM (15). Alpha oscillations might be crucial for protecting the WM (16) against distractors as alpha oscillations in parieto-occipital brain regions in healthy human subjects were found to increase in anticipation of distractors and were directly correlated with increased performance in a WM task (2). Furthermore, phase-locked alpha oscillations have also been found to be associated with enhanced WM encoding and maintenance (2) with higher phase locking reported for better WM accuracy (17). Interestingly, Haenschel and colleagues (18) demonstrated that in schizophrenia, this neural mechanism seems to be disturbed and was associated with poorer WM encoding. 
Therefore, applying a WM paradigm designed to also induce predictable distracting information, could allow us to assess whether ARMS patients demonstrate impaired WM performance partially due to disrupted alpha oscillations in anticipation of distractors. If so, these findings could point to a crucial mechanism in the pathogenesis of psychosis and could add to the prediction of psychosis when combined with other important predictors.

We thus aimed to investigate whether ARMS individuals demonstrate the mechanisms involving alpha oscillations that would allow for the suppression of anticipated obtrusive information. We used single trial analyses of high-density EEG recordings during a modified Sternberg task (2) (Figure 1A) in which participants could anticipate the strength and exact timing of distractors. This allowed us to investigate, on a single trial level, whether compared to healthy controls (HCs), ARMS patients demonstrate decreased alpha oscillations prior to distractors which would be associated with lower performance as indexed by response accuracy and reaction-time (i.e., suggesting an inability to protect their WM against distractors). We also assessed whether in ARMS patients, the phase of alpha activity is still increased during encoding (letter presentation) and also associated with reduced task performance.

\section{Methods}

\section{Setting and Recruitment}

Our sample included 18 ARMS individuals and $21 \mathrm{HCs}$. All participants were recruited as part of the Basel $\boldsymbol{F}$ rüherkennung von $\boldsymbol{P s y c h o s e n}(\boldsymbol{F e P s y})$ project, a prospective multilevel study aiming to improve the early detection of psychosis $(19,20)$. The study was approved by the Ethics committee of Northwestern and Central Switzerland. All participants provided written informed consent. Patients recruited for this study were help-seeking consecutive referrals to the $\boldsymbol{F e P s y}$ Clinic at the University Psychiatric Clinics Basel, which was specifically set up to identify, assess, and treat individuals in the early stages of psychosis. Most participants were referred to the early detection clinic via the University of Basel Psychiatric Clinics or psychiatrists in private practice. 
Some individuals were also referred from other physicians including general practitioners or came on their own due to extensive campaigns (See 20 for study design). HC were recruited from trade schools, hospital staff, and through advertisements. Inclusion criteria for the healthy participants were: no history of psychiatric or neurological disease, no past or present substance abuse or head trauma. More details regarding neurological assessments of ARMS patients have been described elsewhere $(21,22)$.

\section{Sample Description}

Demographic and clinical characteristics are shown in Table 1. 18 ARMS patients $(26.1+/-5.45$ years, 15 males) did not differ from the 21 HCs (25.5 +/- 3.95 years, 15 males $)$ in terms of age, sex, years of education, non-verbal intelligence (LPS) and verbal intelligence (MWT-A). s

\section{Screening Procedure}

The Basel Screening Instrument for Psychosis (BSIP) (23) was applied to identify ARMS individuals. The BSIP is largely based on the PACE inclusion/exclusion criteria (24) and has been shown to have a high predictive validity and a good interrater reliability (23). Exclusion criteria for patients were age $<18$ years, insufficient knowledge of German, low intelligence (IQ $<70$ ), previous episode of schizophrenic psychosis treated with antipsychotics, psychosis clearly due to organic reasons or substance abuse, or psychotic symptoms within a clearly diagnosed affective psychosis or borderline personality disorder.

\section{Assessment of Positive and Negative Psychotic Symptoms}

The Brief Psychiatric Rating Scale Expanded (BPRS-E) $(25,26)$ was used to assess positive and negative psychotic symptoms. The positive psychotic symptom scale was based on the four items hallucinations, suspiciousness, unusual thought content, and conceptual disorganization and the negative psychotic symptom scale was based on the items blunted affect, psychomotor retardation and emotional withdrawal, as defined by Velligan et al. (27). We additionally assessed negative symptoms with the Scale for the Assessment of Negative Symptoms (SANS) (28). 


\section{EEG Task, Recordings and Analyses}

We presented a modified version of the Sternberg task described in more detail by Bonnefond and Colleagues (2) (Figure 1A). We modified the timing of the stimuli of the task as follows: following a fixation dot of $1500 \mathrm{~ms}$, a set of four consonants was serially displayed at a rate of $40 \mathrm{~ms}$ with a blank screen of $1100 \mathrm{~ms}$ between each letter. After the fourth blank screen, either a "weak" (a symbol) or "strong" (a consonant) distractor was presented for 40ms. Participants indicated by button press whether a subsequently appearing letter (known as the probe, the letter following the distractor) was part of the previous memory set ("old" or "new"). The participants could anticipate the distractor type because the weak and strong distractors were grouped in 2 sequential blocks resulting in 120 trials for each condition (i.e., 240 trials in total). The order of the distractors was randomized across individuals. Participants were explicitly asked to answer as fast as possible and to ignore the distractors. A short training session was performed before the actual recording to familiarize the subjects with the task.

Electroencephalography (EEG) recordings were made using BioSemi ActiveTwo electrode system with 64 scalp electrodes (BioSemi, Amsterdam, Netherlands) and digitized at $2048 \mathrm{~Hz}$. Offline EEG data were resampled at $512 \mathrm{~Hz}$ with a high-pass filter of $.5 \mathrm{~Hz}$ to attenuate channel drifts. Bad channels were interpolated using spherical splines, and eye movements and blinks were removed by applying the extended infomax independent component analysis algorithm. EEG data were low-pass filtered at $40 \mathrm{~Hz}$. All trials were segmented separately from -5000 to $1000 \mathrm{~ms}$ relative to stimulus onset (strong and weak distractors). Segments with activity exceeding $\pm 80 \mu \mathrm{V}$ and gradients of $30 \mu \mathrm{V} / \mathrm{s}$ were excluded from further analysis before averaging and rereferencing to the average reference. Brain activities were obtained from 2 regions of interest (ROI) of 5 symmetrical electrodes that revealed highest average amplitudes across all trials and groups on both the posterior left and right area (Figure 2A). The highest amplitude means the maximum averaged amplitude across trials for all subjects at time -5000 to $1000 \mathrm{~ms}$. This is a 
common data driven procedure, for instance see $(29,30)$. The posterior left ROI consisted of electrodes P3, P5, P7, PO3 and PO7 and the posterior right ROI consisted of electrodes P4, P6, P8, PO4, PO8 (Figure 2B).

\section{Wavelet and Intertrial Phase Locking Analyses}

We examined oscillatory activities by means of wavelet decomposition. To calculate intertrial phase locking and single trial amplitude at $1 \mathrm{~Hz}$ frequency steps between 1 and $30 \mathrm{~Hz}$, signals $\mathrm{x}(\mathrm{t})$ from each trial were convolved with a family of complex Morlet wavelets, $w\left(t, f_{0}\right)=A \exp (-$ $\left.t^{2} / 2 \sigma_{t}^{2}\right) \exp \left(2 i \pi f_{0} t\right)$, where $f_{o}$ is the central frequency, $t$ is the time, and $i$ is the imaginary part $(i=\sqrt{ }$ $(-1))$. Wavelets were normalized using the factor $A(w)=\left(\sigma_{t} \sqrt{ }(\pi)\right)^{-1 / 2}$. The family ratio, $m=f_{o} / \sigma_{f}=$ 5 , was used, where $\sigma_{\mathrm{f}}$ is the width of the Gaussian shape in the frequency domain. This ratio was used to optimize the trade-off between the temporal and frequency resolutions of the wavelet convolution (31). Amplitudes for each trial were subsequently averaged over single trials separately for all conditions. Single trial pre-distractor alpha activity $[8-12 \mathrm{~Hz}]$ was extracted from wavelets analyses from $400 \mathrm{~ms}$ before the distractor for a total of $500 \mathrm{~ms}$.

To quantify the extent of normalized inter-trial phase variability across specific electrodes (32), the phase-locking value (PLV) was computed across trials for each electrode, at each time point $(\mathrm{t})$, and frequency $\left(\mathrm{f}_{\mathrm{o}}\right.$ ) yielding a PLV measure that ranges from completely randomized phases across sites $(\mathrm{PLV}=0)$ at a specific time point to a consistent phase across electrodes $(\mathrm{PLV}=1)$. Cumulated PLV is the sum of intertrial PLV elicited during the presentation of the four letters, that is, for each individual, we extracted the highest peak at -4560 to $-3990,-3420$ to $-2850,-2280$ to -1710 and -1140 to -570 .

\section{Statistical Analyses}

Due the repeated measurement present in the data, the statistical analyses were performed using Generalized Linear Mixed effects models (GLME, for binary outcomes) and Linear Mixed Effects models (LME). We chose these models instead of the traditionally used repeated measures 
ANOVA, because they allow analyzing the data on a single trial level. Moreover, they can handle missing data more effectively and are more efficient, parsimonious, and flexible (33-35). Specific factors and models are defined in the Results section. P-values in LME models were estimated using Satterthwaite's approximation (36). Firstly, we assessed group differences by the number of correct responses $(\mathrm{CR})$ in relation to distractor types on a single trial level. Secondly, we assessed how distractor type is related to reaction time (RT) across groups. Thirdly, we investigated the role of alpha amplitude (single trial pre-distractor alpha activity [8-12Hz] obtained from wavelets analyses from $400 \mathrm{~ms}$ before the distractor for a total of $500 \mathrm{~ms}$ ) in protecting the WM against distractors by assessing the relationship between alpha activity and the probability of CR for both distractor types. This was also repeated for RT on correct responses. Fourthly, we assessed the role of intertrial PLV in the alpha band $(8-12 \mathrm{~Hz}$, elicited during the presentation of each of the four letters) on RT. This was also repeated for alpha amplitude.

\section{Results}

Behavioral Analyses

A mixed effects logistic regression model using correct vs. incorrect as binary dependent variable, distractor type and group along with their interaction as fixed effects factors, and per subject randomly varying intercepts and distractor type slopes revealed a significant main effect of distractor type $(\mathrm{p}<0.036)$. That is, the strong distractor resulted in a lower probability of CR compared to the weak distractor independent of group (Figure 1B). The same model, but with log-transformed RT as dependent variable and a Gaussian error distribution, revealed significant main effects of distractor type $(p<0.001)$ and group $(p=0.017)$ and a significant distractor type $\times$ group interaction $(\mathrm{p}=0.050)$. Subgroup analyses revealed that distractor type was only significant for ARMS ( $p=0.002)$ and not for $\mathrm{HC}(\mathrm{p}=0.220)$, indicating that strong distractors resulted in slower RT only in ARMS (Figure 1B). 
An LME model with single trials pre-distractor alpha amplitude as dependent variable, group and distractor type along with their interactions as independent variables and per subject randomly varying intercepts and distractor type slopes, revealed no significant main effect of group, distractor or interaction effects $(\mathrm{p}=0.293, \mathrm{p}=0.971, \mathrm{p}=0.934)$. These results show that the magnitude of pre-distractor alpha amplitude did not differ across groups.

The PLV for different time points, frequencies, groups and distractor types are displayed in Figure 3A. An LME model fitted with cumulated PLV during the letter presentation as dependent variable revealed a trend-level main effect of distractor type $(\mathrm{p}=0.084)$ indicating that PLV was stronger on a trend-level after strong distractors compared to weak distractors. A same model with cumulated alpha amplitude during letter presentation, instead of cumulated alpha PLV, during letter presentation yielded no significant main effect of distractor type $(p=0.533)$, group $(p=0.573)$ or their interaction effect $(\mathrm{p}=0.884)$. These results suggest that during letter presentation, when expecting different distractor types, alpha PLV and not alpha amplitude are modulated differently in HCs only.

\section{Single Trial Pre-distractor Alpha Activity and their Association with Behavioral Performance}

A mixed effects logistic regression model using correct vs. incorrect as binary dependent variable, with pre-distractor alpha activity, distractor type and group along with their interactions as independent variables and per subject randomly varying intercepts and distractor type slopes revealed a significant main effect of distractor type $(\mathrm{p}=0.036)$, and an interaction effect of predistractor alpha activity $\times$ group $(\mathrm{p}=0.009)$ (Figure $2 \mathrm{C})$. These results illustrate that in $\mathrm{HCs}$ increased performance is strongly associated with increased pre-distractor alpha activity. Subgroup analyses revealed that this interaction was due to a significant main effects of predistractor alpha activity in HCs $(p=0.005)$ and not in ARMS patients $(p=0.527)$.

An analogous LME models with log-transformed RT with a Gaussian error distribution revealed significant main effects of pre-distractor alpha activity $(p<.001)$, distractor type $(p<0.001)$, group 
$(p=0.018)$, and interaction effects of pre-distractor alpha activity $\times$ distractor type $(p<0.001)$ (Figure 2D). These results illustrate that pre-distractor alpha activity is associated with increased performance in RT across groups for strong distractors only. Sub-distractor analyses revealed that this interaction was due to a significant main effect of pre-distractor alpha activity in strong distractor $(\mathrm{p}<0.001)$ and not in weak ones $(\mathrm{p}=0.348)$.

Intertrial PLV and Alpha Amplitude during letter presentation and Behavioral Performance

A LME model using correct number of responses as dependent variable, cumulated intertrial alpha PLV during the presentation of each 4 letters, group and distractor type as independent variables along with their interactions and random intercepts per subjects, revealed a significant main effect of PLV ( $p=0.011)$ and a significant effect of group $(\mathrm{p}=0.021)$ (Figure 3B). A similar model with alpha amplitude instead of alpha PLV during letter presentation yielded no significant main effect of alpha amplitude $(p=0.771)$. That is, number of correct responses increased with increasing PLV, and not alpha amplitude, across groups.

The same model, but with log-transformed RT as dependent variable revealed a significant main effect of PLV ( $\mathrm{p}=0.006)$ and an almost significant effect of distractor type $(\mathrm{p}=0.055)$ (Figure 3C). A similar model with alpha amplitude instead of alpha PLV during letter presentation yielded no significant main effect of alpha amplitude $(\mathrm{p}=0.831)$. That is, RT decreased with increasing PLV across groups. Taken together, these results suggest that, across groups, increased alpha PLV elicited during letter presentation is associated with a higher performance as indexed by response accuracy and RT.

\section{Discussion}

The present study was designed to investigate whether the mechanisms involved in suppressing anticipated obtrusive information are still functioning in ARMS patients. In a task where participants could anticipate the strength and exact timing of distractors, we observed that across 
groups, strong distractors reduced WM accuracy. However, only ARMS patients required more processing time for strong distractors compared to weak distractors, which suggests that they are impaired in shielding WM against distractors. On the neural level, parieto-occipital alpha oscillations immediately before distractor presentations seem to be a crucial mechanism for shielding WM against distractors, a process which we hypothesized to be disrupted in ARMS patients. In support of this hypothesis, increased pre-distractor alpha amplitudes at parietooccipital electrode sites were associated with enhanced processing speed for strong distractors across groups. However only in HCs was this activity associated with increased probability of correct responses. Unlike these disruptions during the time-range immediately before distractor presentation, during the encoding of the four letters, increased alpha phase locking values (PLV) was associated with increased WM performance across groups, suggesting that both groups show similar encoding processes in WM.

Converging evidence suggests that alpha oscillations are important for suppressing irrelevant information, leading to enhanced working memory performance $(2,37,38)$. Regarding the role of alpha activity in the functional inhibition of sensory regions, converging studies reported that when attention is allocated to one hemifield, alpha activity increases and decreases on the ipsilateral (i.e., the corresponding brain hemisphere) and contralateral side of the visual system, respectively $(38,39)$ and are associated with performance (6) Furthermore, various findings have revealed that the human brain is capable of discriminating fine temporal durations on the order of $30 \mathrm{~ms}$ or less (40-42), with intracranial recordings in non-human primates demonstrating that alpha oscillations modulate strong inhibitory influence on neural firing selectively at specific phases, which predicted discrimination performance (43). In particular, the timing and amplitude of alpha oscillations seem to be largely important in shielding the WM against distractors (2). In line with these findings, we revealed that increased pre-distractor alpha amplitude was associated with increased correct responses and decreased RT in HCs. ARMS patients showed increased RT 
for strong distractors and a lack of association between pre-distractor alpha amplitudes and probability of correct responses, suggesting that the WM in ARMS patients could be less shielded against irrelevant information. As a consequence, irrelevant information that is filtered-out by the healthy brain would be more deeply engrained and processed in ARMS patient (as reflected by a significant increase in RT in ARMS patients particularly for strong distractors). Although differences in processing speed (RT) could be observed in previous studies assessing patients with schizophrenia in several cognitive tasks (44-46), similar findings are unclear in ARMS patients. That is, while generally lower task performance has been revealed in ARMS patients, processing speed differences in WM task are inconsistent (see (47) and (48) for relevant meta-analyses). It seems that the stronger distractor is more intrusive for WM performance in ARMS patients (as reflected by a significant group*distractor-type interaction), which could already be a sign of poor WM shielding or preservation commonly found in frank psychosis (49).

In addition to investigating the protective effects of alpha oscillations against intrusive stimuli, we have also assessed whether alpha oscillations are phase-synchronized, i.e., timed adequately during letter presentation. Alpha PLV is vital for visual information encoding in $\operatorname{WM}(17,38)$, and have been found to be disturbed in schizophrenia along with associated cognitive deficits (18). In our study, increased PLV during letter presentation was associated with increased performance (i.e., increased correct responses and decreased mean RT) across both groups. Thus, in contrast to previous studies in schizophrenia, ARMS patients appear to still be capable of synchronizing and timing the phase of alpha oscillations during encoding.

Taken together, these results suggest that pre-distractor alpha amplitude and alpha PLV during letter presentations are associated with the preservation and encoding of WM leading to increased performance. In ARMS however, pre-distractor alpha activity was not associated with an increase in performance, suggesting an apparent inability to generate effective alpha activity when 
expecting distractors, leading to a fragile WM preservation. However, PLV of alpha oscillations during letter presentation suggest that their encoding capabilities are still intact.

\section{Limitations}

Noteworthy, differences in performance across groups cannot be attributed to different IQ as no differences were revealed in neither verbal nor nonverbal IQ tests (Table 1). However, the number of ARMS patients in this study is limited as these patients are relatively rare. Many of them only seek help when they have already developed frank psychosis; they are also reluctant to participate in scientific studies as they are quite suspicious at the beginning of the disease. Moreover, 5 of the 18 patients were medicated using antidepressants, which could have influenced the recorded brain activity. Thus, more studies are needed to replicate these findings using more patients and also investigate whether these findings can be confirmed using alternate experimental paradigms.

\section{Conclusions}

In a complex world in which ARMS patients are constantly bombarded with irrelevant stimuli, the lack of proper shielding mechanisms against distractors could lead to defective WM preservation, which could result in substantial confusion that may contribute to the formation of psychotic symptoms. These identified brain activity abnormalities could help to improve the prediction of psychosis and give new insights into the pathophysiological processes leading to the disease. Thus, they may help complement recent efforts targeted at early psychosis detection using multivariate pattern recognition techniques based on neural oscillations $(50,51)$.

\section{Acknowledgements}

The authors would like to thank Ulrike Heitz, Stephanie Menghini and Martina Uttinger for recruiting the patients. We would also like to thank the patients and volunteers for participating in this study. This study was supported by the Swiss National Science Foundation (grant numbers: P0BSP1-152074, 3200-057216.99, 3200-0572216.99, PBBSB-106936, 3232BO-119382). 


\section{References}

1. Zanto TP, Gazzaley A. Neural suppression of irrelevant information underlies optimal working memory performance. The Journal of Neuroscience. 2009;29(10):3059-66.

2. Bonnefond $M$, Jensen $\mathrm{O}$. Alpha oscillations serve to protect working memory maintenance against anticipated distracters. Current biology. 2012;22(20):1969-74.

3. Jensen $\mathrm{O}$, Mazaheri A. Shaping functional architecture by oscillatory alpha activity: gating by inhibition. Frontiers in human neuroscience. 2010;4.

4. Jensen $\mathrm{O}$, Bonnefond M, VanRullen R. An oscillatory mechanism for prioritizing salient unattended stimuli. Trends in cognitive sciences. 2012;16(4):200-6.

5. Rihs TA, Michel CM, Thut G. Mechanisms of selective inhibition in visual spatial attention are indexed by a-band EEG synchronization. European Journal of Neuroscience. 2007;25(2):603-10.

6. Thut G, Nietzel A, Brandt SA, Pascual-Leone A. a-Band electroencephalographic activity over occipital cortex indexes visuospatial attention bias and predicts visual target detection. The Journal of Neuroscience. 2006;26(37):9494-502.

7. Lakatos P, Karmos G, Mehta AD, Ulbert I, Schroeder CE. Entrainment of neuronal oscillations as a mechanism of attentional selection. science. 2008;320(5872):110-3.

8. Haegens S, Osipova D, Oostenveld R, Jensen O. Somatosensory working memory performance in humans depends on both engagement and disengagement of regions in a distributed network. Human brain mapping. 2010;31(1):26-35.

9. Wilsch A, Henry MJ, Herrmann B, Maess B, Obleser J. Alpha oscillatory dynamics index temporal expectation benefits in working memory. Cerebral Cortex. 2014:bhu004.

10. Forbes N, Carrick L, Mclntosh A, Lawrie S. Working memory in schizophrenia: a meta-analysis. Psychological medicine. 2009;39(06):889-905.

11. Clementz BA, Geyer MA, Braff DL. Poor P50 suppression among schizophrenia patients and their first-degree biological relatives. American Journal of Psychiatry. 2014.

12. Schultze-Lutter F, Michel C, Schmidt S, Schimmelmann B, Maric N, Salokangas R, et al. EPA guidance on the early detection of clinical high risk states of psychoses. European Psychiatry. 2015;30(3):405-16.

13. Fusar-Poli P, Bechdolf A, Taylor MJ, Bonoldi I, Carpenter WT, Yung AR, et al. At risk for schizophrenic or affective psychoses? A meta-analysis of DSM/ICD diagnostic outcomes in individuals at high clinical risk. Schizophrenia bulletin. 2013;39(4):923-32.

14. Bodatsch M, Brockhaus-Dumke A, Klosterkötter J, Ruhrmann S. Forecasting Psychosis by EventRelated Potentials—Systematic Review and Specific Meta-Analysis. Biol Psychiatry. 2015;77(11):951-8.

15. Anticevic A, Repovs G, Corlett PR, Barch DM. Negative and nonemotional interference with visual working memory in schizophrenia. Biol Psychiatry. 2011;70(12):1159-68.

16. Roux F, Uhlhaas PJ. Working memory and neural oscillations: alpha-gamma versus theta-gamma codes for distinct WM information? Trends in cognitive sciences. 2014;18(1):16-25. 
17. Freunberger R, Fellinger R, Sauseng P, Gruber W, Klimesch W. Dissociation between phase-locked and nonphase-locked alpha oscillations in a working memory task. Human brain mapping. 2009;30(10):3417-25.

18. Haenschel C, Linden DE, Bittner RA, Singer W, Hanslmayr S. Alpha phase locking predicts residual working memory performance in schizophrenia. Biological psychiatry. 2010;68(7):595-8.

19. Riecher-Rössler A, Pflueger MO, Aston J, Borgwardt SJ, Brewer WJ, Gschwandtner U, et al. Efficacy of using cognitive status in predicting psychosis: a 7-year follow-up. Biol Psychiatry. 2009;66(11):1023-30.

20. Riecher-Rössler A, Gschwandtner U, Aston J, Borgwardt S, Drewe M, Fuhr P, et al. The Basel early-detection-of-psychosis (FEPSY)-study-design and preliminary results. Acta Psychiatrica Scandinavica. 2007;115(2):114-25.

21. Riecher-Rössler A, Pflueger MO, Aston J, Borgwardt SJ, Brewer WJ, Gschwandtner U, et al. Efficacy of using cognitive status in predicting psychosis: a 7-year follow-up. Biological psychiatry. 2009;66(11):1023-30.

22. Ittig S, Studerus E, Papmeyer M, Uttinger M, Koranyi S, Ramyead A, et al. Sex differences in cognitive functioning in at-risk mental state for psychosis, first episode psychosis and healthy control subjects. European Psychiatry. 2015;30(2):242-50.

23. Riecher-Rössler A, Aston J, Ventura J, Merlo M, Borgwardt S, Gschwandtner U, et al. [The Basel Screening Instrument for Psychosis (BSIP): development, structure, reliability and validity]. Fortschr Neurol Psychiatr. 2008;76(4):207-16.

24. Yung AR, Phillips LJ, McGorry PD, McFarlane CA, Francey S, Harrigan S, et al. Prediction of psychosis. A step towards indicated prevention of schizophrenia. Br J Psychiatry Suppl. 1998;172(33):1420.

25. Ventura J, Green MF, Shaner A, Liberman RP. Training and quality assurance with the Brief Psychiatric Rating Scale:" the drift busters.". International Journal of Methods in Psychiatric Research. 1993.

26. Lukoff D, Nuechterlein K, Ventura J. Manual for the expanded brief psychiatric rating scale. Schizophr Bull. 1986;12:594-602.

27. Velligan D, Prihoda T, Dennehy E, Biggs M, Shores-Wilson K, Crismon ML, et al. Brief psychiatric rating scale expanded version: How do new items affect factor structure? Psychiatry research. $2005 ; 135(3): 217-28$.

28. Andreasen NC. Scale for the Assessment of Negative Symptoms (SANS). The British Journal of Psychiatry. 1989.

29. Kometer M, Schmidt A, Bachmann R, Studerus E, Seifritz E, Vollenweider FX. Psilocybin biases facial recognition, goal-directed behavior, and mood state toward positive relative to negative emotions through different serotonergic subreceptors. Biol Psychiatry. 2012;72(11):898-906.

30. Dias EC, Butler PD, Hoptman MJ, Javitt DC. Early sensory contributions to contextual encoding deficits in schizophrenia. Arch Gen Psychiatry. 2011;68(7):654-64.

31. Kometer M, Schmidt A, Jancke L, Vollenweider FX. Activation of Serotonin 2A Receptors Underlies the Psilocybin-Induced Effects on alpha Oscillations, N170 Visual-Evoked Potentials, and Visual Hallucinations. J Neurosci. 2013;33(25):10544-51.

32. Lachaux J-P, Rodriguez E, Martinerie J, Varela FJ. Measuring phase synchrony in brain signals. Human brain mapping. 1999;8(4):194-208. 
33. Bagiella E, Sloan RP, Heitjan DF. Mixed-effects models in psychophysiology. Psychophysiology. 2000;37(01):13-20.

34. Dixon P. Models of accuracy in repeated-measures designs. Journal of Memory and Language. 2008;59(4):447-56.

35. Gueorguieva R, Krystal JH. Move over anova: Progress in analyzing repeated-measures data andits reflection in papers published in the archives of general psychiatry. Archives of general psychiatry. 2004;61(3):310-7.

36. Satterthwaite FE. An approximate distribution of estimates of variance components. Biometrics bulletin. 1946:110-4.

37. Händel BF, Haarmeier T, Jensen O. Alpha oscillations correlate with the successful inhibition of unattended stimuli. Journal of cognitive neuroscience. 2011;23(9):2494-502.

38. Klimesch W, Sauseng P, HansImayr S. EEG alpha oscillations: the inhibition-timing hypothesis. Brain research reviews. 2007;53(1):63-88.

39. Jensen $\mathrm{O}$, Mazaheri A. Shaping functional architecture by oscillatory alpha activity: gating by inhibition. Frontiers in human neuroscience. 2010;4:186.

40. Nobre AC, O'Reilly J. Time is of the essence. Trends in cognitive sciences. 2004;8(9):387-9.

41. Nobre AC, Correa A, Coull JT. The hazards of time. Current opinion in neurobiology. 2007;17(4):465-70.

42. McAuley JD, Jones MR. Modeling effects of rhythmic context on perceived duration: a comparison of interval and entrainment approaches to short-interval timing. Journal of Experimental Psychology: Human Perception and Performance. 2003;29(6):1102.

43. Haegens S, Nácher V, Luna R, Romo R, Jensen O. a-Oscillations in the monkey sensorimotor network influence discrimination performance by rhythmical inhibition of neuronal spiking. Proceedings of the National Academy of Sciences. 2011;108(48):19377-82.

44. Driesen NR, Leung H-C, Calhoun VD, Constable RT, Gueorguieva R, Hoffman R, et al. Impairment of working memory maintenance and response in schizophrenia: functional magnetic resonance imaging evidence. Biological psychiatry. 2008;64(12):1026-34.

45. Schlösser RG, Koch K, Wagner G, Nenadic I, Roebel M, Schachtzabel C, et al. Inefficient executive cognitive control in schizophrenia is preceded by altered functional activation during information encoding: an fMRI study. Neuropsychologia. 2008;46(1):336-47.

46. Johnson MR, Morris NA, Astur RS, Calhoun VD, Mathalon DH, Kiehl KA, et al. A functional magnetic resonance imaging study of working memory abnormalities in schizophrenia. Biological psychiatry. 2006;60(1):11-21.

47. Fusar-Poli P, Deste G, Smieskova R, Barlati S, Yung AR, Howes O, et al. Cognitive functioning in prodromal psychosis: a meta-analysis. Archives of general psychiatry. 2012;69(6):562-71.

48. Bora E, Murray RM. Meta-analysis of cognitive deficits in ultra-high risk to psychosis and firstepisode psychosis: do the cognitive deficits progress over, or after, the onset of psychosis? Schizophrenia bulletin. 2013:sbt085.

49. Fatouros-Bergman H, Cervenka S, Flyckt L, Edman G, Farde L. Meta-analysis of cognitive performance in drug-naïve patients with schizophrenia. Schizophrenia research. 2014;158(1):156-62.

50. Ramyead A, Kometer M, Studerus E, Koranyi S, Ittig S, Gschwandtner U, et al. Aberrant Current Source-Density and Lagged Phase Synchronization of Neural Oscillations as Markers for Emerging Psychosis. Schizophr Bull. 2014. 
Ramyead et al., 2016

51. Ramyead A, Studerus E, Kometer M, Uttinger M, Gschwandtner U, Fuhr P, et al. Prediction of psychosis using neural oscillations and machine learning in neuroleptic-naïve at-risk patients. World J Biol Psychiatry. 2015:1-11. 


\section{Figure legends}

Figure 1: (A) The modified Sternberg task. Following a fixation dot of $1500 \mathrm{~ms}$, four consonants were sequentially presented at a rate of $40 \mathrm{~ms}$ per letter. Between each of these four letters, a blank picture was presented for $1100 \mathrm{~ms}$. In the retention interval, either a "weak" (a symbol) or "strong"' (a consonant) distractor was presented. Subjects indicated by button press whether the probe (the letter following the distractor) was part of the memory set ("old" or "new"). Participants could anticipate the distractor type as the weak and strong distractors were grouped in 4 blocks resulting in 120 trials for each condition (i.e., 240 trials in total). The order of the distractors was randomized across individuals.

(B) The hit rate and reactions times in response to the memory probe for each group. Although across both groups a lower correct response rate was observed for the strong distractor, the response times were significantly longer for the strong in patients only. This indicates in ARMS patients, strong distractors seem to be encoded more strongly in their working memory and interferes with retrieval processes. Error bars correspond to the $95 \%$ confidence interval.

Figure 2: (A) Time-course of amplitude values for the strong and weak distractor condition and across groups $(1-30 \mathrm{~Hz})$. Red dashed lines represent the timing of each letter being presented and black dashed lines represent the timing of the distractors. (B) The scalp map depicting the electrodes studies in the current study. (C) Pre-distractor alpha amplitude as a function of reaction time across groups. (D) Pre-distractor alpha amplitude as a function of probability of correct response across groups. Error bars correspond to the 95\% confidence interval.Figure 3: (A) Timecourse of alpha phase-locking values for the strong and weak distractor conditions and across groups $(1-30 \mathrm{~Hz})$. Black dashed lines represent the timing of the distractors. (B) Phase-locking values elicited during letter encoding (4 letters) as a function of number of correct response for each group (C) Phase-locking values elicited during letter encoding (4 letters) and reaction time for each group. Error bars correspond to the $95 \%$ confidence interval. 

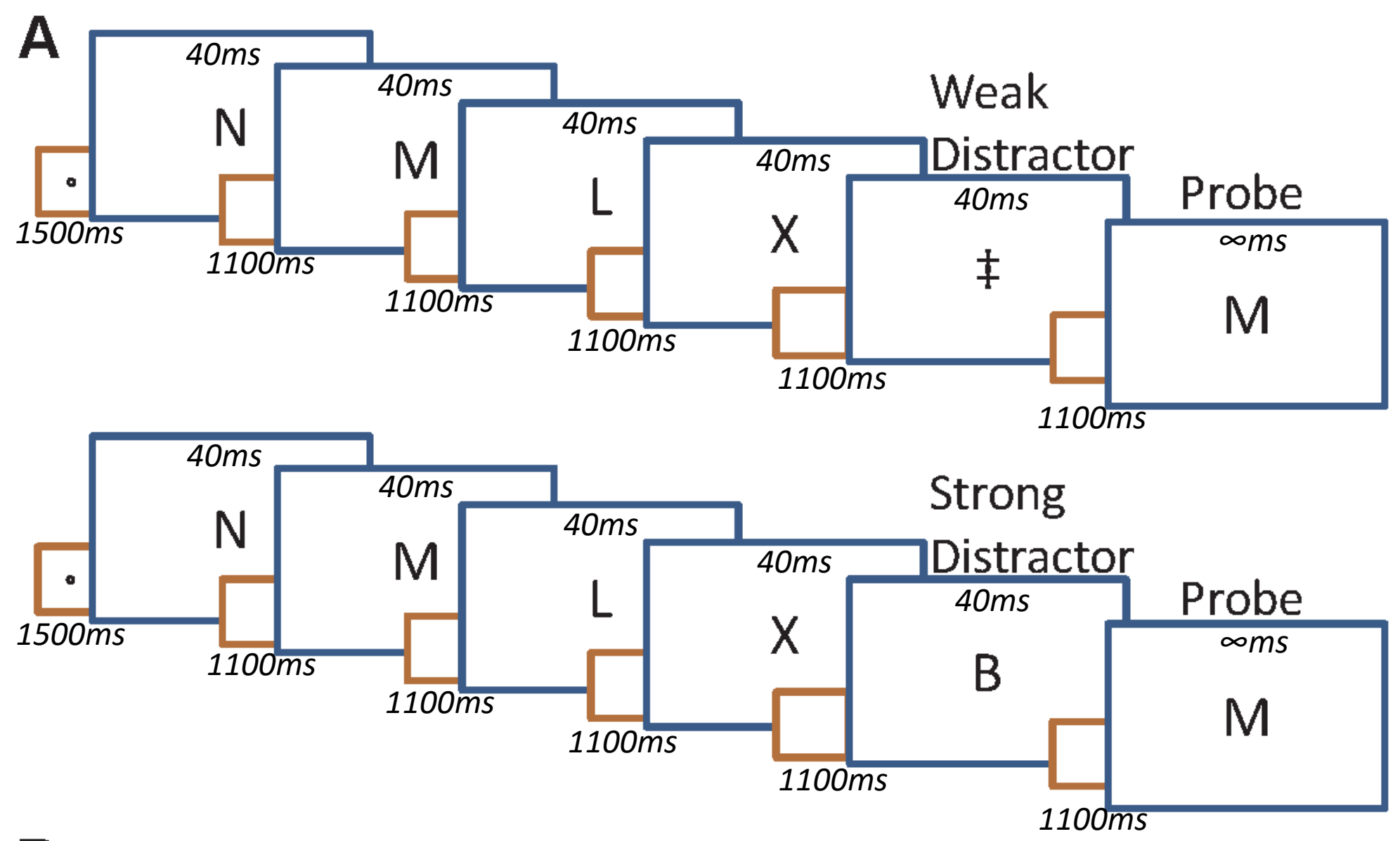

B
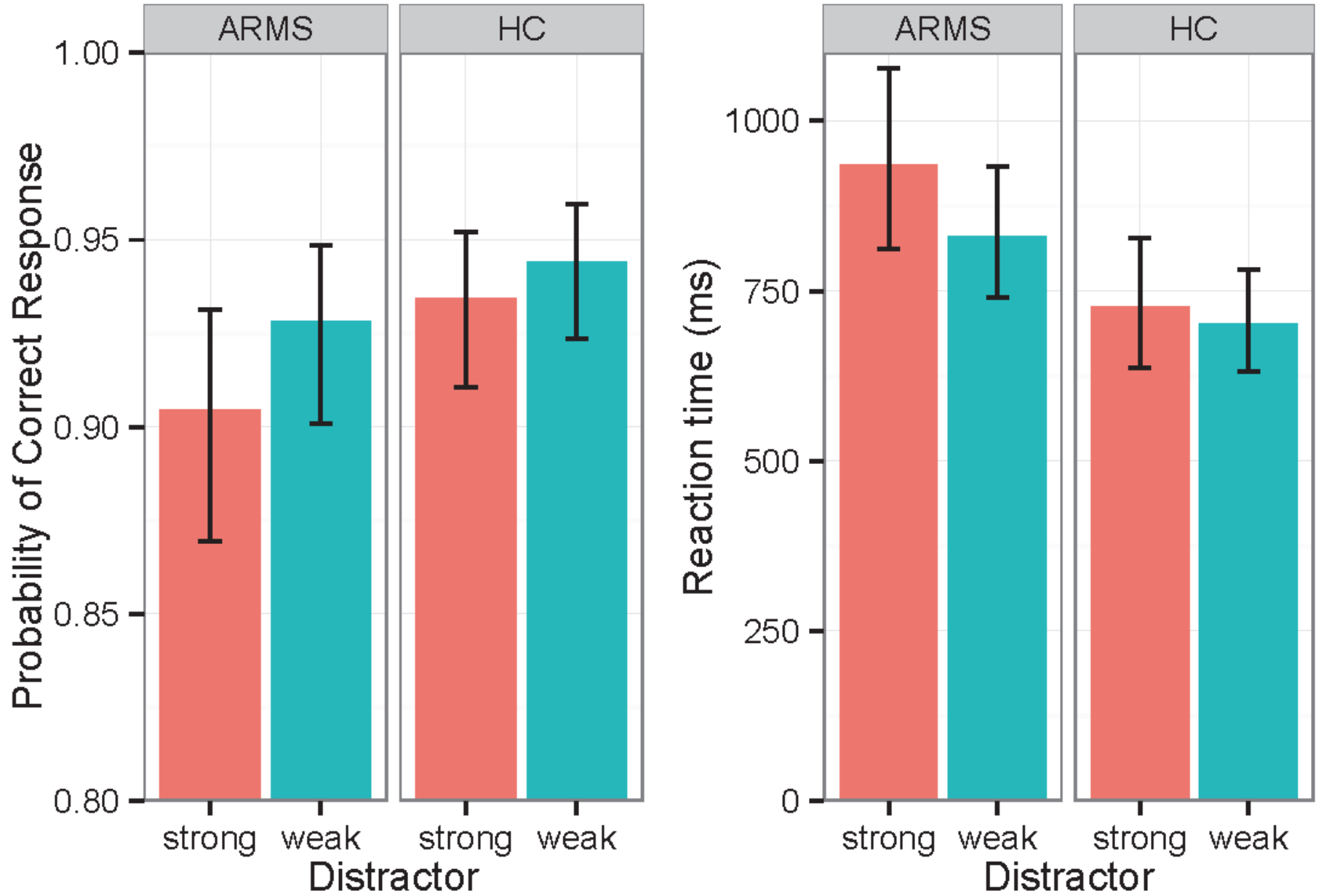

strong weak 

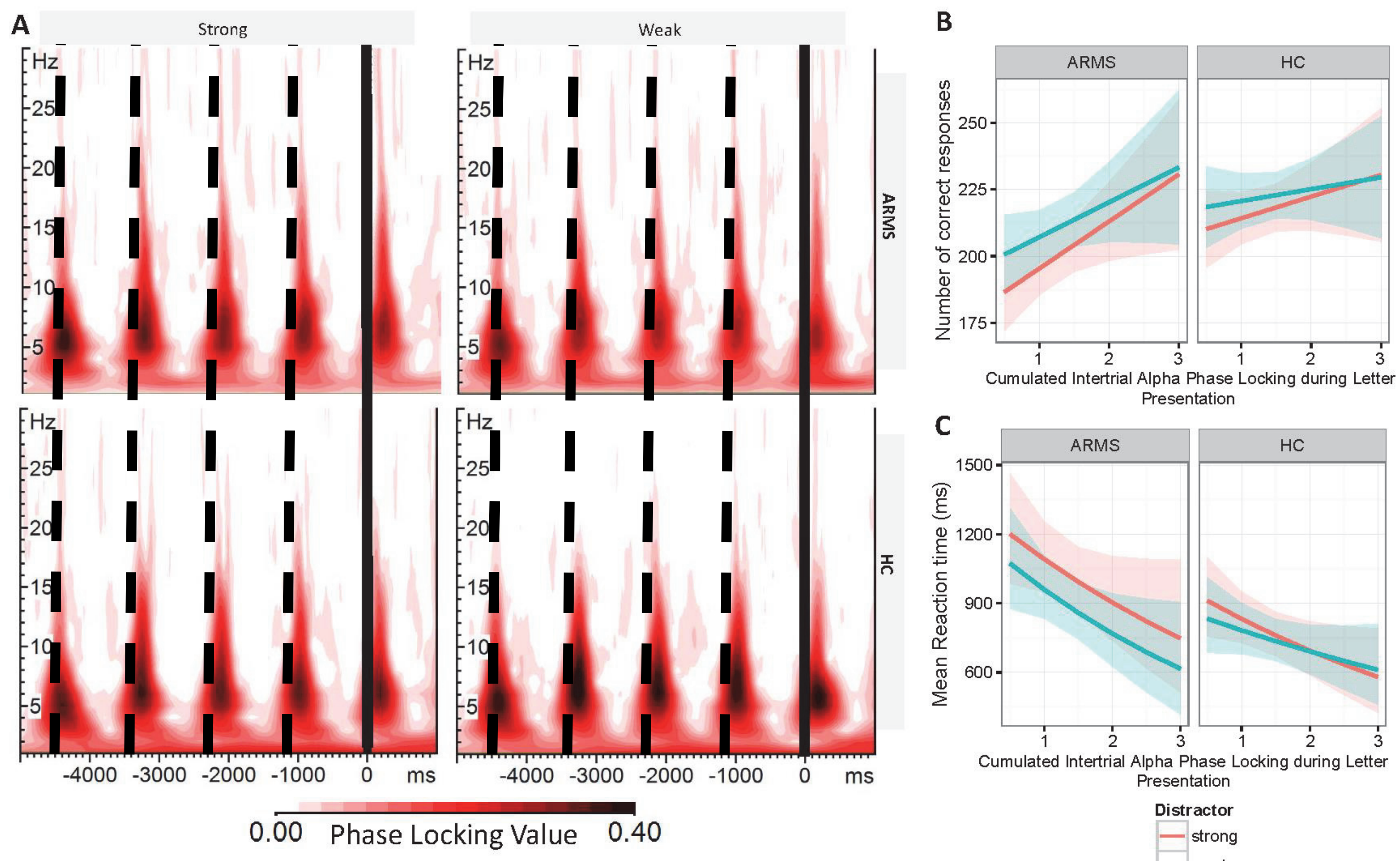

Cumulated Intertrial Alpha Phase Locking during Letter Presentation

Distractor - strong — weak 
Table 1: Demographic and Clinical Characteristics

\begin{tabular}{|c|c|c|c|}
\hline & $\begin{array}{c}\mathrm{HC} \\
\mathrm{N}=21\end{array}$ & $\begin{array}{l}\text { ARMS } \\
\mathrm{N}=18\end{array}$ & $P$ Value \\
\hline Gender: & & & 0.464 \\
\hline Female & $6(28.6 \%)$ & $3(16.7 \%)$ & \\
\hline Male & $15(71.4 \%)$ & $15(83.3 \%)$ & \\
\hline Age & $25.5(3.95)$ & $26.1(5.45)$ & 0.738 \\
\hline Years of education: & & & 0.603 \\
\hline$<9$ years & $0(0.00 \%)$ & $2(11.8 \%)$ & \\
\hline $9-11$ years & $5(35.7 \%)$ & $4(23.5 \%)$ & \\
\hline $12-13$ years & $5(35.7 \%)$ & $8(47.1 \%)$ & \\
\hline $14-20$ years & $4(28.6 \%)$ & $3(17.6 \%)$ & \\
\hline BPRS total score & & $38.9(10.8)$ & \\
\hline BPRS Positive Symptoms & & $6.47(1.97)$ & \\
\hline BPRS Negative Symptoms & & $5.88(2.50)$ & \\
\hline SANS total score & & $17.5(15.2)$ & \\
\hline LPS & $120(13.5)$ & $114(11.1)$ & 0.143 \\
\hline MWT-A & $113(12.2)$ & $114(14.2)$ & 0.869 \\
\hline Antipsychotics currently & & $0(0 \%)$ & \\
\hline \multicolumn{4}{|l|}{ Antidepressants currently: } \\
\hline No & & $12(70.6 \%)$ & \\
\hline Yes & & $5(29.4 \%)$ & \\
\hline Anxiolytics currently: & & $0(0 \%)$ & \\
\hline
\end{tabular}

$\mathrm{HC}=$ healthy controls, $\mathrm{ARMS}=$ at-risk mental state patients; BPRS = Brief Psychiatric Rating Scale; SANS = Scale for the Assessment of Negative Symptoms; LPS = Leistungsprüfsystem; MWT-A = Mehrfachwahl-Wortschatz-Test. Categorical and continuous variables were compared by Chi-square tests and ANOVAs, respectively. Numbers in brackets indicate SD for continuous variables and percentages for categorical variables. 